\title{
Adaptaciones al Subsistema Nacional de Evaluación de Costa Rica a partir de los ODS
}

\author{
The National Evaluation Subsystem of Costa Rica: \\ adaptations based on the SDGs
}

\section{Eddy García Serrano ${ }^{I}$}

I Máster Iberoamericano en Evaluación de Políticas Públicas (Universidad Internacional de Andalucía, España). Licenciado en Ciencias Políticas (Universidad de Costa Rica, UCR). Docente en programas de maestría en el Instituto Centroamericano de Administración Pública (ICAP) y programas de especialización en el Centro de Investigación y Capacitación en Administración Pública (CICAPUCR). Jefe de la Unidad de Evaluación del Ministerio de Planificación Nacional y Política Económica de Costa Rica.

凶·eddy.garcia@mideplan.go.cr https://orcid.org/0000-0001-

5307-4052

\section{Resumen}

Este artículo se presenta bajo un corte institucional, con una recopilación de los avances en el subsistema de evaluación en el sector público costarricense en materia de evaluación de intervenciones públicas, con énfasis particula en la ilustración de elementos estratégicos que se han configurado en los últimos años desde el Ministerio de Planificación Nacional y Política Económica como ente rector en evaluación, teniendo como referencia y punto de encuentro los postulados de la Agenda 2030 y el marco de los Objetivos de Desarrollo Sostenible. Se realiza un recorrido sobre los instrumentos nacionales que rigen la función de evaluación y han sido permeados por este marco internacional, y se reseña un caso concreto. Se concluye con un conjunto de reflexiones que aspiran servir de insumo para alimentar el debate sobre la necesidad de profundizar los esquemas de evaluación en el aparato estatal.

Palabras clave: evaluación, administración pública, Costa Rica, desarrollo sostenible

\section{Abstract}

The article compiles the advances in the sub-system of evaluation in the field of public interventions evaluation in the Costa Rican public sector. It particularly 
emphasizes the strategic elements recently configured by the Ministry of National Planning and Economic Policy as the governing body in evaluation, bearing in mind the postulates of the 2030 Agenda and the Sustainable Development Goals' framework as a reference and meeting point. In this sense, a tour around the national governing instruments for evaluation that have been permeated by this international framework is made, as well as a review of a specific case. It concludes with a series of reflections to feed the debate on the need to step forward in the state apparatus' evaluation schemes. Keywords: evaluation, public administration, Costa Rica, sustainable development.

\section{Ensayo}

Dentro del sector público costarricense la función de evaluación se encuentra consagrada por una serie de normas que van desde la Constitución Política, en su artículo 11; la Ley 5525 de Planificación (Costa Rica. Asamblea Legislativa, 2012), la Ley 8131 sobre Presupuestos Públicos (Costa Rica. Asamblea Legislativa, 2001); hasta los decretos ejecutivos 35755, 37735 (Costa Rica. Poder Ejecutivo, 2010, 2013). Dicho marco designa al Ministerio de Planificación Nacional y Política Económica (Mideplan) como rector del Sistema Nacional de Planificación (SNP) y del Subsistema Nacional de Seguimiento y de Evaluación (SINE). Este último ente busca promover la capacidad gerencial del sector público, y se propone:

1. Desarrollar una cultura de seguimiento y evaluación en el sector público

2. Elaborar y ejecutar estrategias, lineamientos, metodologías y procedimientos de seguimiento y evaluación [...] del sector público.

3. Asesorar, promover y regular los procesos de seguimiento y evaluación.

4. Establecer agendas anuales de evaluaciones estratégicas en las instituciones.

5. Retroalimentar a las y los ministros rectores y jerarcas institucionales sobre los resultados de las evaluaciones.

Mideplan ha venido realizando acciones de innovación de gestión pública en diferentes campos bajo la orientación del enfoque de gestión para resultados en el desarrollo, y en especial se recalcan los decididos esfuerzos para promover un esquema riguroso de evaluación que incentive la cultura evaluativa en el sector público (Ministerio de Planificación Nacional y Política Económica [Mideplan], 2018a). Tomando en consideración el marco que imponen la Agenda 2030 y los Objetivos de Desarrollo Sostenible (ODS) a los países, el presente artículo aspira a exponer sintéticamente los avances de Costa Rica en torno a la emisión de la Política Nacional de Evaluación 2018-2030 (PNE, https://www.mideplan.go.cr/politica-nacional-de-evaluacion-pne) y la configuración 
de la Agenda Nacional de Evaluaciones (ANE, https://www.mideplan.go.cr/agenda-nacional-de-evaluaciones) dentro de los planes nacionales de desarrollo (PND); lo anterior como ejemplo de adaptación de los aparatos institucionales ( o de los diferentes instrumentos de planificación nacional) bajo los principios de la Agenda 2030.

A partir de estos dos puntos de referencia se aspira a reflejar la experiencia de Costa Rica adaptando elementos normativos, instrumentales y prácticos de la función de evaluación de intervenciones públicas hacia los propósitos de los ODS.

\section{Política Nacional de Evaluación 2019-2030 y ODS}

La PNE aspira a direccionar en el corto, mediano y largo plazo la función de evaluación dentro del SNP, SINE y las instancias que lo integran. Ello expresa el compromiso de Mideplan de mejorar el diseño, implementación y resultados de las intervenciones públicas, así como la transparencia y rendición de cuentas por medio de un sistema de evaluación sistemático, integral y creíble, lo que respalda el mandato legal asignado como ente rector así como las prioridades institucionales en dicha materia. Su objetivo es contribuir a la mejora de la gestión pública al emplear la evaluación como instrumento para sustentar la toma de decisiones, el aprendizaje, el uso racional de los recursos públicos. Como aspiración inicial, la conformación de la PNE se propuso:

1. Involucrar de manera participativa a diversos actores estratégicos de Costa Rica en el proceso de construcción de una política nacional de evaluación, para favorecer su implementación y uso.

2. Fortalecer el rol de la evaluación en la toma de decisiones políticas y en la sociedad costarricense.

3. Definir y consensuar los roles de los distintos actores en los procesos de evaluación.

Se destina principalmente a las instituciones, entes y organizaciones integrantes del Sistema Nacional de Planificación que se encuentran vinculadas al ciclo de gestión de las intervenciones públicas. Además, pretende servir de material de referencia para diversos actores interesados en la materia como el sector descentralizado y autónomo, la academia, organizaciones no gubernamentales, evaluadores(as) y la sociedad civil en general, entre otros.

Esta política establece como uno de sus enfoques la Agenda 2030, al indicar que «los procesos de evaluación se deben basar en los principios de desarrollo sostenible, los cuales evidenciarán los avances y brechas en la implementación de intervenciones públicas» (Mideplan, 2018b, p. 14). Para operacionalizar dicho enfoque se identifican 
dos mecanismos centrales: i) inclusión de una línea de acción denominada «Incorporar los compromisos país en el ciclo de la gestión pública en el corto, mediano y largo plazo». En la figura 1 se destaca el contenido de esta línea de acción; valga decir que las actividades cuentan con una serie de indicadores, metas y plazos específicos para garantizar su medición en el tiempo y su trazabilidad.

Figura 1. Vínculos entre PNE y los ODS

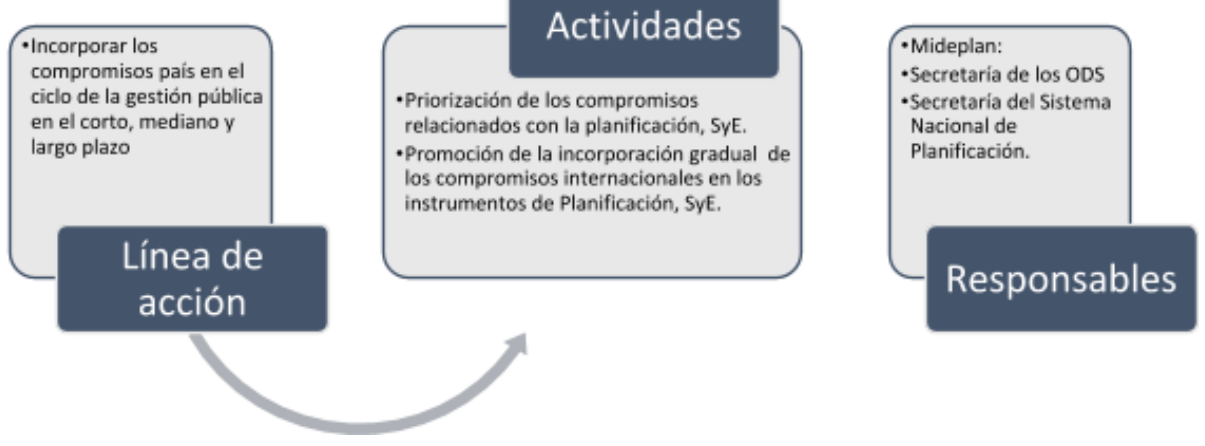

Fuente: Elaboración propia a partir de Mideplan (2018b).

En segunda instancia se ha configurado todo un eje dentro de la política denominado «Participación en evaluación», cuyo objetivo es «fortalecer la integración y participación de diversos actores en procesos evaluativos». Incluye una gama de actividades para su cumplimiento, las cuales apuntan acuerpar el lema de No dejar a nadie atrás dentro del desarrollo de procesos evaluativos.

Resalta la figura de la Plataforma Nacional de Evaluación como mecanismo de implementación de la política:

[...] se constituye un espacio abierto de participación de actores relacionados con el desarrollo de cultura de la evaluación en el país, está dirigida por Mideplan, con la participación de instituciones del Gobierno central, academia, redes de evaluadores, Asamblea Legislativa, Contraloría General de la República, Defensoría de los Habitantes, sociedad civil organizada, socios cooperantes internacionales. (Mideplan, 2018b, p. 72)

Todos los actores que participan en la Plataforma tienen el derecho a ser consultados, a participar (en distintos niveles) en los procesos de evaluación y a acceder a la 
información sobre la implementación de las recomendaciones generadas tras los resultados de las evaluaciones.

\section{Agenda Nacional de Evaluaciones 2019-2022}

La ANE es la herramienta que agrupa el conjunto de intervenciones públicas con carácter estratégico que son evaluadas en el periodo de vigencia del PND. El Gobierno de la República en el periodo 2019-2022 respalda la generación de evaluaciones a las intervenciones en el máximo nivel de planificación, cristalizando la ANE dentro del PNDIP, y manteniendo la meta prevista de ejecutar 15 evaluaciones en el periodo, lo cual es representativo en un contexto de ajuste fiscal y restricciones presupuestarias dentro del sector público.

Dicha agenda es multisectorial e integra — para el periodo gubernamental vigente- 15 intervenciones públicas de carácter estratégico, las cuales se fundamentan en una «valoración sistemática sobre el diseño, la ejecución y los resultados de políticas, planes, programas y proyectos con base en un conjunto de criterios de valor preestablecidos» (Mideplan, 2017, p. 23.). Esta valoración genera recomendaciones basadas en evidencias para apoyar la toma de decisiones y mejorar la gestión pública; a la vez genera información para elevar el debate y la discusión nacional sobre la legitimidad de la acción pública para resolver o mitigar las problemáticas sociales.

Con respecto a la toma de decisiones fundamentada o basada en evidencia, la Organización para la Cooperación y el Desarrollo Económico (OCDE) ha enfatizado que «las buenas prácticas de gobernanza sugieren que las políticas deberían basarse en evidencia sólida derivada de análisis rigurosos de los hechos disponibles sobre el asunto que debe atender la política, siendo la evaluación un mecanismo idóneo para esos propósitos» (2016, p. 60). Aspectos claves que se han integrado a la configuración del proceso de evaluación en Costa Rica, el cual se estructura en etapas: programación, diseño, ejecución y uso.

La puesta en marcha de procesos de evaluación se realiza de manera consciente, organizada y con una intencionalidad expresa para valorar un aspecto de las intervenciones públicas (objeto de evaluación) y de esta manera contribuir a un mejor desempeño de estas. Cualquier evaluación comienza con un propósito, que responde a la diversidad de necesidades de información que manifiestan los actores involucrados con la intervención pública bajo análisis; por tal razón, la incorporación de dichas consideraciones en todo el proceso evaluativo garantiza un mayor aprovechamiento y uso de los resultados de la evaluación. 
Figura 2. Proceso de evaluación

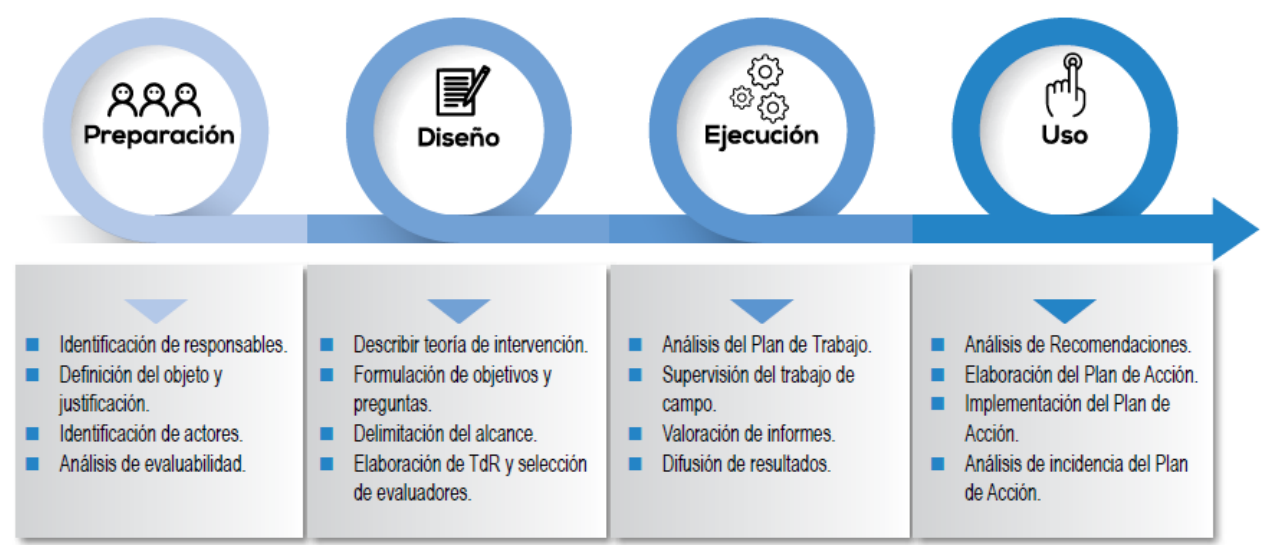

Fuente: Mideplan 2017.

Desde la metodología de construcción de la ANE (Mideplan, 2018c), y para responder a la pregunta ¿qué se va a evaluar? se decidió incorporar, entre los criterios de selección y priorización de intervenciones públicas, la vinculación de estas intervenciones con los ODS. Específicamente, el criterio vinculación ODS consistió en analizar el grado de vinculación entre la intervención pública propuesta para evaluar y los objetivos y metas ODS, de forma tal que aquellas intervenciones que estuvieran relacionadas a una mayor cantidad de objetivos y metas ODS se le otorgaba un puntaje más alto y, con ello, un aumento en la posibilidad de ser seleccionada para la conformación de la ANE.

En este sentido, cada evaluación ejecutada en el marco de la ANE tiene identificados los ODS a los que apuntan dichas intervenciones. Por ejemplo, el ODS 8 (Trabajo decente y crecimiento económico) y el ODS 6 (Igualdad de género), son los que en mayor medida se presentan. En la figura 3 se refleja en detalle este aspecto.

Una vez que se ha definido qué se va a evaluar, se desarrolla el proceso de evaluación propiamente, en el cual la perspectiva ODS también se encuentra presente mediante la incorporación de preguntas de evaluación dirigidas a generar evidencia de las contribuciones de la intervención evaluada con los ODS a los que esta se encuentra alineada. Un caso particular que muestra esta relación es una evaluación ejecutada en el sector Ambiente y Energía, que se presenta a continuación. 
Figura 3. Frecuencia de ODS en la ANE

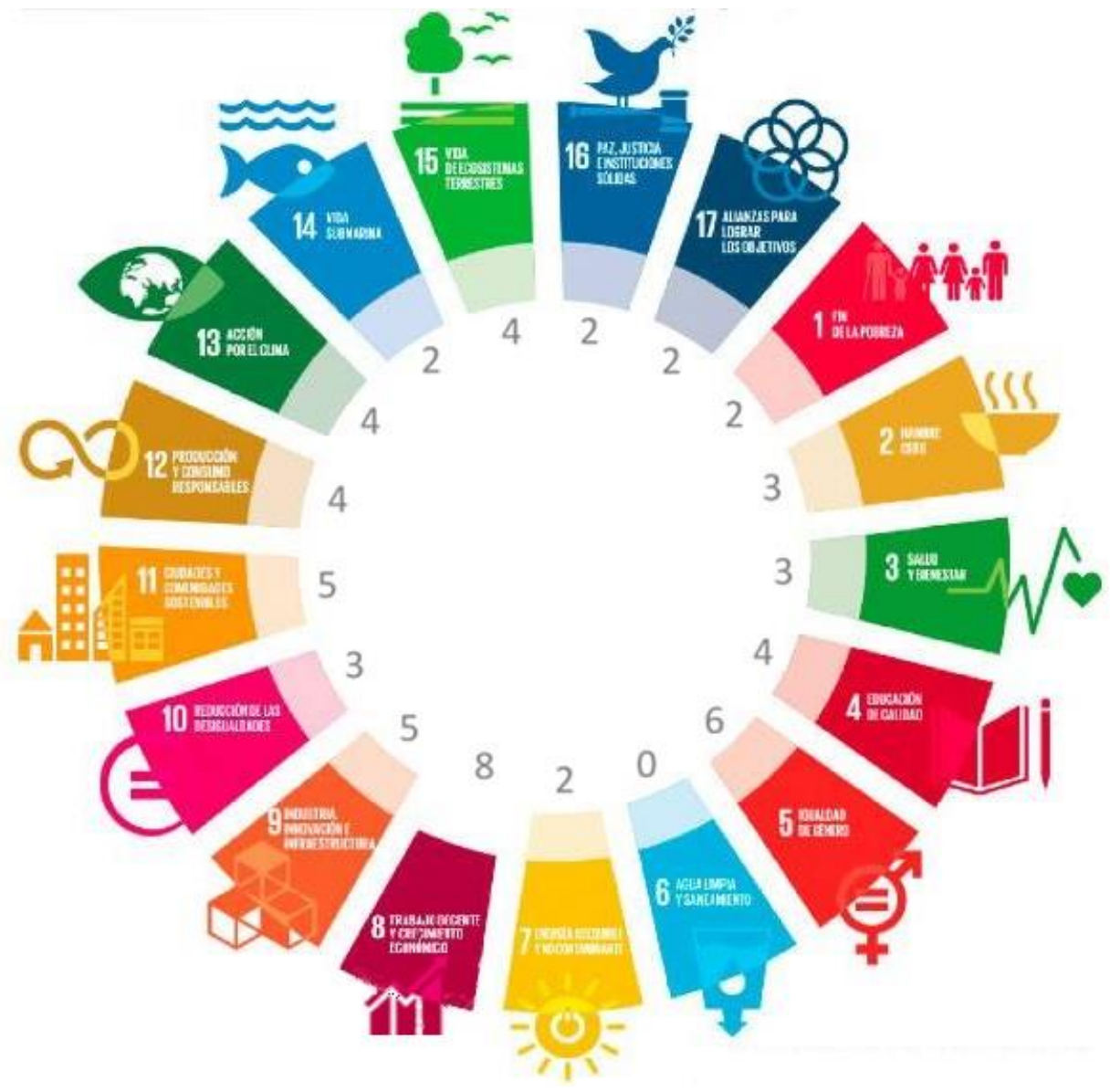

Fuente: Informe Voluntario de ODS 2017-2020, Mideplan (2020c)

\section{Evaluación al Plan Nacional de Desarrollo Forestal 2010-2020}

El PNDF 2011-2020 es un instrumento estratégico que tiene como misión «asegurar la implementación y consolidación de la política forestal del país, que contribuya al desarrollo sostenible en su dimensión social, económica y ambiental» (Ministerio de Ambiente Energía y Telecomunicaciones [MINAET], 2011, p. 9). Representa el 
instrumento oficial de planificación para uso, manejo y protección de los recursos forestales del país, en el cual se establecen directrices y lineamientos a desarrollar en el territorio nacional; está compuesto por una política general, 12 políticas forestales del Estado y siete ejes estratégicos, los cuales son considerados desafíos claves para el sector forestal y constituyen su columna vertebral; también cuenta con una estrategia de implementación y una estrategia de seguimiento. Es operativizada por el Sistema Nacional de Áreas de Conservación (SINAC), el Fondo Nacional de Financiamiento Forestal (FONAFIFO), la Oficina Nacional Forestal (ONF) y el Colegio de Ingenieros Agrónomos (CIAGRO).

La evaluación al plan valora el diseño, la implementación y los resultados alcanzados hasta 2019; con la evidencia obtenida se aspira a orientar la toma de decisiones en la formulación del nuevo PNDF 2021-2030.

Para el abordaje de los mencionados propósitos se diseñó una evaluación de tipo formativa, que considerara tanto el diseño del PNDF como su implementación y el avance en el logro de resultados. Además, se trabajó con un equipo de evaluación externo, que estuvo compuesto por representantes de la Unidad de Evaluación de Mideplan y por una evaluadora joven emergente contratada por el proyecto FOCELAC (Fomento de Capacidades y Articulación de Actores de la Evaluación en América Latina). También, se contó con apoyo de representantes de SINAC, FONAFIFO, ONF, CIAGRO, la Secretaría de Planificación Sectorial (SEPLASA) y el Despacho del Viceministerio de Ambiente.

Metodológicamente se desarrolló una estrategia metodológica mixta, con un abordaje predominantemente cualitativo, incorporando técnicas cuantitativas para fortalecer el diseño y la triangulación de los datos, lo que aumentó, a su vez, la credibilidad y la coherencia de la información. Como indica Bamberger (2012), los métodos mixtos favorecen la complementariedad de las técnicas, de manera tal que se aprovechan las ventajas de ambos tipos de métodos. En otras palabras, con el uso de métodos mixtos es posible utilizar técnicas de recolección de información cualitativa que permiten contextualizar y explicar los procesos de interés en la evaluación; con el abordaje cuantitativo se complementa la información, con opciones de descripción e, inclusive, según el diseño, de generalización de la información a cierto grupo poblacional.

En el caso de esta evaluación en particular, la predominancia de métodos cualitativos responde a la necesidad de profundizar en el análisis de la experiencia del Plan actual como un proceso, más allá de los datos estadísticos sobre los resultados, es decir, interesa comprender los mecanismos y estrategias que funcionaron o no a lo largo del proceso, de manera que se puedan extraer aprendizajes para la elaboración e implementación del futuro PNDF 2021-2030.

Dentro del criterio de sostenibilidad, se conformó la pregunta ¿qué elementos del diseño e implementación deben reforzarse en un próximo PNDF? Y, ligada a esta, una 
subpregunta: ¿cómo se articula el PNDF con los ODS correspondientes? Para estudiar esta vinculación se examinaron las metas establecidas para cada uno de los 17 ODS y se compararon con los resultados esperados de cada uno de los siete ejes del PNDF. Para analizar la vinculación se estableció la siguiente rúbrica de cuatro niveles:

1. Nula $=$ No existe vinculación.

2. Baja $=$ Existe vinculación directa del PNDF con una meta de los ODS.

3. Media = Existe vinculación directa del PNDF con dos metas de los ODS.

4. Alta $=$ Existe vinculación directa del PNDF con más de tres metas de los ODS.

En el caso particular de los ODS 13 y 15, por ejemplo, la relación alta se destaca con una serie de indicadores que tienen una relación directa y que muestran avances importantes en el país. Esto se destaca como punto positivo, ya que a pesar de que por razones de temporalidad el PNDF no fue construido en función de los ODS, se pueden identificar puntos de convergencia en sus metas (Mideplan 2020a, p. 73).

Figura 4. Vinculación de los ejes del PNDF con los ODS

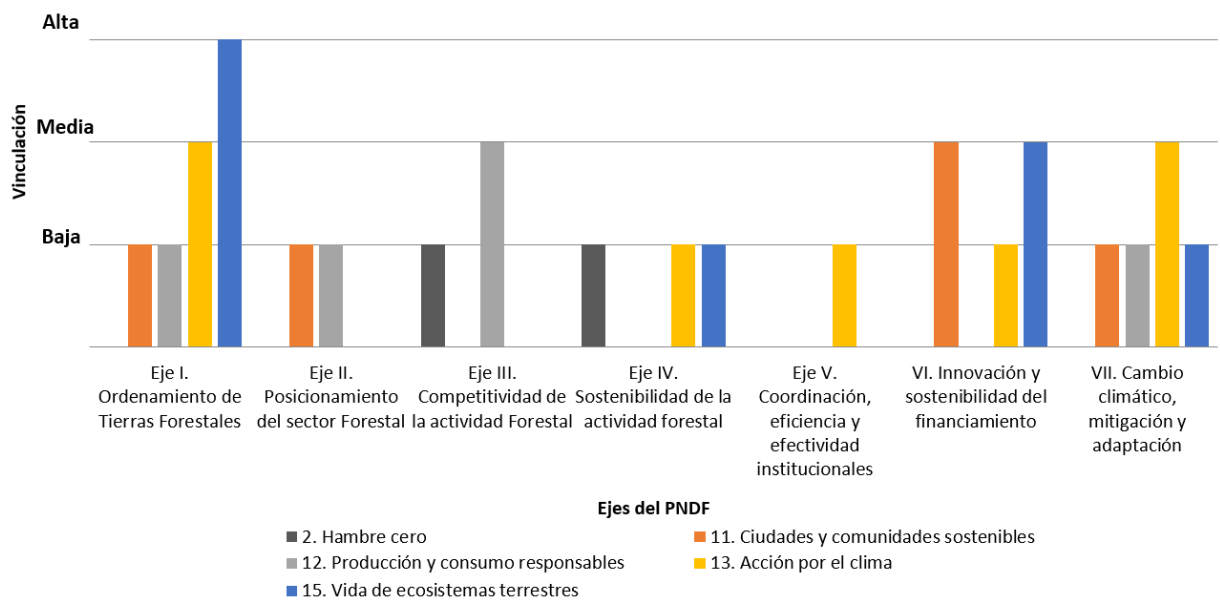

Fuente: Mideplan (2020a).

Como resultado del análisis se logró determinar que el eje 1 «Ordenamiento de tierras forestales» tiene una vinculación alta con el ODS 15 «Vida y ecosistemas terrestres» y una vinculación media con el ODS 13 «Acción por el clima». Esto destaca como elemento positivo, ya que dicho ODS tiene como objetivo la gestión sostenible de los bosques, la lucha contra la desertificación y la pérdida de biodiversidad, elementos que afectan al país y para los cuales son necesarias acciones. Actualmente, el país ha 
recuperado más del $20 \%$ de su cobertura forestal en los últimos 25 años. De modo sintético, la evaluación concluye que:

Existe una conciencia entre los actores consultados de la importancia de poder vincular un próximo PNDF con los instrumentos de política pública, [...] y compromisos asumidos a nivel internacional. Es importante para la construcción del próximo PNDF que se pueda realizar un análisis exhaustivo de cada una de las metas de los ODS y diversos instrumentos, de forma que durante el proceso de construcción del PNDF se puedan entrelazar las metas y objetivos de este con los acuerdos internacionales y nacionales ya preestablecidos. (Mideplan, 2020a, p. 70)

De esta manera queda reflejado que los resultados de la evaluación sitúan en los actores consultados las discusiones sobre los ODS y el debate, lo que fundamenta para la toma de decisiones elementos validados para orientar la política pública.

Los hallazgos considerados en la evaluación y el prisma de análisis sobre ODS para actualizar los instrumentos de planificación con base en esta información visualizan un escenario favorable para la consideración de los resultados de la evaluación dentro del nuevo PNDF a formular. Los compromisos institucionales apuntan al desarrollo de actividades en esta línea, por tanto, queda constancia de esta manera que la evaluación se pone al servicio de alimentar un nuevo ciclo de planificación, añadiendo los puntos de mejora y optimización acontecidos; esta descripción tan sucinta como emblemática refleja el espíritu de la evaluación como herramienta de mejora.

La ANE contempla el principio de la participación en los procesos de evaluación de las intervenciones públicas que la integran, de cara a promover una buena gobernanza, y logra potenciar el diálogo e interacción entre los distintos actores del desarrollo (representantes institucionales, sociedad civil, beneficiarios de los bienes y servicios, entre otros), ya que estos son considerados como fuentes de información durante el proceso evaluativo y agentes receptores de los resultados de las evaluaciones. Esto conlleva un replanteamiento de la forma tradicional de concebir la acción pública, direccionada hacia la apertura e inclusividad, así como efectiva en la protección de los derechos y libertades civiles y políticos de la población, y genera sinergias para obtener resultados de desarrollo y el fortalecimiento del esquema democrático en el país.

Cabe resaltar que en un reciente estudio del Ministerio se determinó, sobre la participación en evaluaciones, que:

La adopción de esquemas de evaluación con participación amplia de diversos actores es incipiente (un 50\% de los actores consultados indica que no o no sabe si se incorporarán en el proceso a los beneficiarios de las intervenciones), lo que implica asignar un rol más protagónico y el involucramiento de los actores 
beneficiarios considerando sus necesidades y participando de forma sistemática en el proceso de evaluación. (Mideplan, 2020b, p. 24)

Entre las buenas prácticas respecto a la integración de la población en varios de los procesos de evaluación en el marco de la ANE, según la etapa del proceso, se resaltan los siguientes:

1. Programación: durante esta etapa se realiza un mapeo exhaustivo de las diferentes poblaciones que están relacionadas con la intervención y en consecuencia lo estarían en la evaluación, analizando cuál sería su rol en la evaluación, expectativas y usos eventuales de la evaluación, visualizando con ello el abanico existente de actores y cuándo y dónde serían involucrados en el proceso de evaluación.

2. Diseño: la incorporación de la participación de la ciudadanía organizada en la elaboración o validación de objetivos y preguntas de evaluación relacionadas con sus intereses y usos eventuales de la evaluación, considerando así los intereses de la población. Suma a esta etapa la reconstrucción de la teoría de la intervención desde la visión y concepción de la población. Esto como prácticas indudables de apropiación de la evaluación por la ciudadanía.

3. Ejecución: La participación de la población beneficiaria como fuente primaria de datos para responder las preguntas de evaluación abre un espacio estratégico de escucha de la voz de la población en cuanto a la valoración de la bondad de la intervención en cuestión; resultan con ello conclusiones y recomendaciones más realistas, precisas y útiles para la gestión pública. Asimismo, la solicitud expresa en los términos de resultados que los resultados de la evaluación sean públicos y difundidos — al menos una vez-a la población que de una u otra forma participa en la intervención pública y la evaluación de esta, ha sido una herramienta de rendición de cuenta del quehacer de la gestión de pública y uso de recursos.

4. Uso: la disposición pública de los planes de acción para realizar las recomendaciones facilita el involucramiento de la población en su rol de control ciudadano y, en ocasiones, facilitando actividades para la puesta marcha de dichas recomendaciones.

\section{Reflexiones finales}

Con la aplicación de esquemas evaluativos se potencia el diálogo e interacción entre los distintos actores del desarrollo (representantes institucionales, sociedad civil, 
beneficiarios de los bienes y servicios, entre otros), ya que estos son considerados como fuentes de información durante el proceso evaluativo. Esto conlleva un replanteo de la forma tradicional de concebir la acción pública, mucho más abierta e inclusiva, y genera sinergias para obtener resultados.

La continuidad y el fortalecimiento de estos esquemas orientan la gestión pública hacia el mejoramiento, oportunidad, calidad y pertinencia en la prestación de los bienes y servicios públicos, asumiendo el prisma que supone la Agenda 2030. Usualmente se le asigna a la evaluación una carga centrada en la perspectiva de rendición de cuentas; no obstante, desde Mideplan, además de esta perspectiva se contempla la dimensión del aprendizaje y la mejora, lo cual es en sí un valor agregado a la hora de desarrollar acciones de evaluación, ya que en el marco de la adopción del enfoque de gestión para resultados en el desarrollo (GpRD) se asume esta tarea como mecanismo para la revisión de lo que se está haciendo, cómo se está haciendo y para qué se está haciendo, síntoma de las entidades y organizaciones con vocación de mejora continua.

De cara a obtener evaluaciones que apunten hacia esos propósitos, idealmente las intervenciones públicas deben contemplar de manera sistemática al me nos tres grandes elementos: en primer lugar, que el origen de la acción pública constituya una reflexión crítica sobre las distintas alternativas disponibles para atender o mitigar las problemáticas presentes en la realidad nacional; en segunda instancia, que la alternativa seleccionada se conceptualice en términos formales siguiendo un esquema de planificación sistemático, bajo el enfoque de gestión para resultados en el desarrollo, e incorpore los elementos básicos que permitan dar seguimiento al avance y consecución de objetivos y resultados; y en último término, la presencia de las condiciones idóneas en el contexto nacional, sectorial e institucional para que su implementación se ejecute de manera óptima.

Contemplando la evaluación como una acción que tiene un carácter público, es importante que esta cuente con las condiciones idóneas para llevarse a cabo, fundamentado en que la acción de evaluar comprende la disposición de recursos, capacidades, esfuerzos articulados y tiempo para su desarrollo y, por tanto, exige una adecuada planificación para garantizar su éxito y calidad. En la implementación de la ANE queda en evidencia que gran parte de las intervenciones no cuentan con las condiciones óptimas de evaluabilidad (entendida como las posibilidades que presenta una intervención pública de ser evaluada) con lo que se limita la medición de resultados y se obliga a voltear la mirada hacia otros tipos de evaluación menos comprehensivos pero que resulten útiles en los contextos donde se desenvuelven.

Bajo estas premisas, en este periodo gubernamental la acción evaluativa ha estado enfocada en maximizar el uso de los recursos en el sentido de establecer las bases para determinar de manera fehaciente las conexiones entre la o las razones que originan una acción pública (problemas o situaciones adversas) y los elementos que permitan 
explicar la manera en que esta resuelve o mitiga dicha problemática. Ello está orientado a verificar la consistencia, lógica y coherencia de las políticas públicas, dimensionar en contexto sus diseños y valorar las estrategias una vez que han sido aplicadas, de cara al correcto funcionamiento de estas para atender sus objetivos y propósitos, incrementado bajo el sello de los ODS, aspecto medular a la hora de analizar los aportes que realizan en concreto al desarrollo del país en su conjunto.

\section{Referencias bibliográficas}

Bamberger, M. (2012). Introduction to mixed methods in impact evaluation. Recuperado de https://www.interaction.org/wp-content/uploads/2019/03/Mixed-Methods-in-Impact-Evaluation-English.pdf

Costa Rica. Asamblea Legislativa. (2001). Ley 8131: Ley de la Administración Financiera de la República y Presupuestos Públicos. Recuperado de http://www.pgrweb.go.cr /scij/Busqueda/Normativa/Normas/nrm_texto_completo.aspx?param1=NRTC\& nValor1=1\&nValor2=47258\&nValor3=73503\&strTipM=TC

Costa Rica. Asamblea Legislativa. (2012). Ley 5525: Ley de Planificación Nacional. Recuperada de http://www.pgrweb.go.cr/scij/Busqueda/Normativa/Normas/ nrm_texto_completo.aspx?param1=NRTC\&nValor1=1\&nValor2=34439\&nValor3 $=91172 \&$ strTipM $=\mathrm{TC}$

Costa Rica. Poder Ejecutivo. (2010). Decreto ejecutivo 35755: Sistema Nacional de Evaluación. Recuperado de http://www.pgrweb.go.cr/scij/Busqueda/Normativa/Normas/nrm_texto_completo.aspx?param1=NRTC\&nValor1=1\&nValor2=67309\&nValor3=79671\&strTipM=TC

Costa Rica. Poder Ejecutivo. (2013). Decreto ejecutivo 37735: Reglamento General del Sistema Nacional de Planificación. Recuperado de http://www.pgrweb.go.cr/scij/ Busqueda/Normativa/Normas/nrm_norma.aspx?param1=NRM\&nValor1=1\& nValor2=75112\&nValor3=118645\&strTipM=FN

Ministerio de Ambiente Energía y Telecomunicaciones. (2011). Plan Nacional de Desarrollo Forestal: 2011-2020. San José, Costa Rica: Comunicaciones Milenio. Recuperado de https://www.sirefor.go.cr/pdfs/tematicas/Politicas_Nacionales/Plan_ Nacional_Desarrollo_Forestal_2011-2020.pdf

Ministerio de Planificación Nacional y Política Económica. (2017). Manual de evaluación para intervenciones públicas. San José, Costa Rica: Mideplan. Recuperado de https://documentos.mideplan.go.cr/share/s/6eepeLCESrKkft6Mf5SToA

Ministerio de Planificación Nacional y Política Económica. (2018a). Plan Nacional de Desarrollo y de Inversión Pública del Bicentenario, 2019-2022. San José, Costa Rica: Mideplan. Recuperado de https://www.mideplan.go.cr/plan-nacional-desarrollo 
Ministerio de Planificación Nacional y Política Económica. (2018b). Política Nacional de Evaluación 2018-2030. San José, Costa Rica: Mideplan. Recuperado de https://www.mideplan.go.cr/politica-nacional-de-evaluacion-pne

Ministerio de Planificación Nacional y Política Económica. (2018c). Metodología Agenda Nacional de Evaluación 2019-2022. San José, Costa Rica: Mideplan. Recuperado de https://documentos.mideplan.go.cr/share/s/KgHjkGABTQyDal4GefLYbA

Ministerio de Planificación Nacional y Política Económica. (2020a). Evaluación de diseño, procesos y resultados: Plan Nacional de Desarrollo Forestal. San José, Costa Rica: Mideplan. Recuperado de https://documentos.mideplan.go.cr/share/ s/xRmBBS_GSOOhCNakLX6M9g

Ministerio de Planificación Nacional y Política Económica. (2020b). Reporte de evaluaciones en el Sistema Nacional de Planificación 2019-2022: características básicas. San José, Costa Rica: Mideplan. Recuperado de https://documentos.mideplan.go.cr/share/s/Cvbh4BMgTRe07KW7Ou-VBg

Ministerio de Planificación Nacional y Política Económica. (2020c). Segundo informe nacional voluntario Objetivos de Desarrollo Sostenible Costa Rica 2020. Desarrollo sostenible en acción: La ruta hacia la sostenibilidad. San José, Costa Rica: Mideplan. Recuperado de https://documentos.mideplan.go.cr/share/s/KnmDPk5cT76rag_g2JclZw

Organización para la Cooperación y el Desarrollo Económicos. (2016). Estudio de gobernanza pública en Costa Rica. Buena gobernanza: De procesos a resultados. Recuperado de https://www.comex.go.cr/media/5228/estudio-sobre-gobernanza-pública-2015.pdf 